\title{
Probability-Dependent Gain-Scheduled Filtering for Stochastic Systems with Missing Measurements
}

\author{
Guoliang Wei, Zidong Wang, Bo Shen and Maozhen Li
}

\begin{abstract}
This paper addresses the gain-scheduled filtering problem for a class of discrete-time systems with missing measurements, nonlinear disturbances and external stochastic noises. The measurement missing phenomenon is assumed to occur in a random way, and the missing probability is time-varying with securable upper and low bounds that can be measured in real time. The multiplicative noise is a state-dependent scalar Gaussian white noise sequence with known variance. The addressed gain-scheduled filtering problem is concerned with the design of a filter such that, for the admissible random measurement missing, nonlinear parameters and external noise disturbances, the error dynamics is exponentially mean-square stable. The desired filter is equipped with time-varying gains based primarily on the timevarying missing probability and is therefore less conservative than the traditional filter with fixed gains. It is shown that the filter parameters can be derived in terms of the measurable probability via the semi-definite programme method.
\end{abstract}

Index Terms-Filtering; missing measurements; gain scheduling; time-varying Bernoulli distribution; probability-dependent Lyapunov functions

\section{INTRODUCTION}

The general idea of filtering problems is to form a kind of "best estimate" for the true value of some certain system based on some potentially noisy observations [1]-[4], [8], [13]-[16]. Filtering problem serves as one of the fundamental problems in the areas of control and signal processing. During the past few decades, the filtering problem has attracted considerable attention and some effective filtering strategies have been exploited in the literature that include Kalman filtering scheme [9] and $H_{\infty}$ filtering methods [10]. The developed filtering approaches have been applied in a variety of systems such as uncertain time-delay systems [10], stochastic systems [11] and nonlinear systems [6], [11].

Copyright (c) 2011 IEEE. Personal use of this material is permitted. However, permission to use this material for any other purposes must be obtained from the IEEE by sending an email to pubs-permissions@ieee.org

This work was supported in part by the Leverhulme Trust of the U.K., the Engineering and Physical Sciences Research Council (EPSRC) of the U.K. under Grant GR/S27658/01, the National Natural Science Foundation of China under Grants 61028008, 61074016 and 60974030, the Shanghai Natural Science Foundation of China under Grant 10ZR1421200, and the Alexander von Humboldt Foundation of Germany.

G. Wei, Z. Wang and B. Shen are with the School of Information Science and Technology, Donghua University, Shanghai 200051, China. Z. Wang is also with the Department of Information Systems and Computing, Brunel University, Uxbridge, Middlesex, UB8 3PH, United Kingdom. (Email: Zidong. Wang@brunel.ac.uk)

$\mathrm{M}$. Li is with the Department of Electronic and Computer Engineering, Brunel University, Uxbridge, Middlesex, UB8 3PH, United Kingdom.
Due to various reasons such as probabilistic network congestion and intermittent mechanical failures, it has been well recognized that the missing measurement phenomenon is inevitable in many real-world systems connected via networks. The filtering problem with missing measurements has recently received renewed research interests due mainly to the popularity of networked control systems [7], [10]. As a simple yet effective model, the Bernoulli distribution model has been utilized in different systems to reflect the missing measurements or packet dropouts, for example, nonlinear systems [12], time-delay systems [10] and networked control systems [12], etc. Unfortunately, in almost all existing literature concerning Bernoulli distribution model, the missing probability has been implicitly assumed to be a fixed constant resulting in a timeinvariant filter structure. In reality, however, for dynamics variation in the environment, the severity of the missing measurement phenomenon might be changeable with time, and therefore the missing probability is often time-varying.

For the filter design problems for time-varying systems, the gain-scheduling approach has been found to be one of the most effective ones, whose main idea is to design filter gains as functions of the scheduling parameters which are supposed to be available in real time. Gain-scheduled filtering problems have been an attractive research focus in the past decade, see e.g. [5]. Associated with the gain-scheduling technique is the utilization of parameter-dependent Lyapunov function with hope to reduce the possible conservatism [1]. Nevertheless, there has been little research attention on the filtering problem for discrete-time nonlinear stochastic systems with randomly missing phenomenon, especially when the time-varying nature comes mainly from the missing probability. This is indeed a challenging topic that deserves much research effort from the community. It is, therefore, the purpose of this paper to make one of the first few attempts to deal with the missingprobability-dependent gain-scheduled filtering problems.

The main contributions of this paper are summarized as follows: 1) a new filtering problem is addressed for a class of discrete-time nonlinear stochastic systems with missing measurements via a gain-scheduling approach; 2) a sequence of stochastic variables satisfying Bernoulli distributions is exploited to reflect the time-varying features of the missing measurements in sensors; 3) a time-varying Lyapunov function dependent on the missing probability is proposed and then applied to improve the performance of the gain-scheduled filters; and 4) the filter parameters can be updated online according to the missing probabilities estimated through statistical tests. 


\section{PRoblem Formulation}

Consider the following class of discrete-time nonlinear stochastic systems:

$$
\begin{aligned}
x(k+1) & =A x(k)+B f(z(k))+D x(k) \omega(k) \\
y_{0}(k) & =C x(k)
\end{aligned}
$$

where $x(k) \in \mathbb{R}^{n}$ is the state, $y_{0}(k) \in \mathbb{R}^{m}$ is the ideal measurement output (without data missing) and $z(k):=Z x(k)$. $\omega(k)$ is an one-dimensional Gaussian white noise sequence satisfying $\mathbb{E}\{\omega(k)\}=0$ and $\mathbb{E}\left\{\omega^{2}(k)\right\}=\sigma^{2} . A, B, C, D$ and $Z$ are constant matrices with appropriate dimensions. $x(0)=\rho$ is the initial state and the output matrix $C$ is assumed to be full of row rank.

The nonlinear vector-valued function $f(\cdot)$ represents the nonlinear disturbance satisfying the following sector-bounded condition with $f(0)=0$ :

$$
\left[f(z(k))-F_{1} z(k)\right]^{T}\left[f(z(k))-F_{2} z(k)\right] \leq 0,
$$

where $F_{1}$ and $F_{2}$ are constant real matrices of appropriate dimensions and $F=F_{2}-F_{1}$ is a symmetric positive definite matrix. It is customary that such nonlinear function $f(\cdot)$ belongs to the sector $\left[F_{1}, F_{2}\right]$ [6]. In this case, the nonlinear function $f(z(k))$ can be decomposed into a linear part and a nonlinear part as

$$
f(z(k))=F_{1} z(k)+f_{s}(z(k)),
$$

and it follows from (3) that

$$
f_{s}^{T}(z(k))\left(f_{s}(z(k))-F z(k)\right) \leq 0 .
$$

The measurement output with sensor data missing is described by

$$
y(k)=\xi(k) y_{0}(k)=\xi(k) C x(k),
$$

where $\xi(k) \in \mathbb{R}$ is a random white sequence characterizing the probabilistic sensor data missing phenomenon, which obeys the following time-varying Bernoulli distribution:

$$
\begin{aligned}
& \operatorname{Prob}\{\xi(k)=1\}=\mathbb{E}\{\xi(k)\}=p(k), \\
& \operatorname{Prob}\{\xi(k)=0\}=1-\mathbb{E}\{\xi(k)\}=1-p(k),
\end{aligned}
$$

where $p(k)$ is a time-varying positive scalar sequence that belongs to $\left[\begin{array}{ll}p_{1} & p_{2}\end{array}\right] \subseteq\left[\begin{array}{ll}0 & 1\end{array}\right]$ with the constant $p_{1}$ and $p_{2}$ being the lower and upper bounds of $p(k)$. In this paper, we assume that $\xi(k), \omega(k)$ and $\rho$ are uncorrelated. Furthermore, the kind of measurements missing that obey the probability distribution law (7) is said to be admissible.

Remark 1: In (6), a random white sequence satisfying the time-varying Bernoulli distribution is introduced to reflect the missing measurement phenomenon that has attracted considerable attention in the past few years, see e.g. [10]. However, the missing probability in most relevant literature has always been assumed to be a constant. Such an assumption, unfortunately, tends to be conservative in handling time-varying missing measurements. In this paper, the missing probability is allowed to be time-varying with known lower and upper bounds which will then be used to schedule filter gains, thereby reducing the possible conservatism.
In this paper, we aim to construct the following probabilitydependent gain-scheduled filter for (1) and (6):

$$
x_{f}(k+1)=G(p(k)) x_{f}(k)+H(p(k)) y(k),
$$

where $x_{f}(k) \in \mathbb{R}^{n}$ is the state estimate and $p(k)$ is the timevarying scheduling parameter taking value in $\left[\begin{array}{ll}p_{1} & p_{2}\end{array}\right] . G(p(k))$ and $H(p(k))$ are the scheduled filter gains of the following structure:

$$
G(p(k))=G_{0}+p(k) G_{f}, \quad H(p(k))=H_{0}+p(k) H_{f}
$$

where $G_{0}, G_{f}, H_{0}$ and $H_{f}$ are the constant filter parameters to be designed and $p(k)$ is the time-varying missing probability that can be estimated/measured via statistical tests in real time.

Remark 2: Different from the conventional filters, the above gain-scheduled filter structure comprises two kinds of filter gains: the constant (fixed) parameters $G_{0}, G_{f}, H_{0}$ and $H_{f}$, and the time-varying parameter $p(k)$. Here, $p(k)$ takes value in the interval $\left[\begin{array}{ll}p_{1} & p_{2}\end{array}\right]$ and can be measured in real time. In certain applications such as the reliability analysis for sensors, if the data missing probability for a particular sensor is greater than 0.5 through statistical tests, then such a sensor would be replaced or at least repaired. In other words, the upper bound for the data missing probability is 0.5 . In this paper, the interval constraint $\left[\begin{array}{ll}p_{1} & p_{2}\end{array}\right]$ is added to reflect such an engineering practice and also facilitate the later analysis. Obviously, with this type of gain-scheduled filters, the conservatism can be reduced since more information about the missing measurement phenomenon is utilized. Note that this kind of gain-scheduling technique has been extensively applied to deal with robust control and filtering problems for uncertain systems with time-varying parameters, see e.g. [5].

Letting $\bar{x}(k)=\left[\begin{array}{ll}x^{T}(k) & x_{f}^{T}(k)\end{array}\right]^{T}$, the error dynamics of the filtering process is derived from (1), (6) and (8) as follows:

$$
\begin{aligned}
\bar{x}(k+1)= & \bar{A}(p(k)) \bar{x}(k)+\bar{B} f(z(k))+(\xi(k)-p(k)) \bar{C}(p(k)) \\
& \times N \bar{x}(k)+\bar{D} N \bar{x}(k) \omega(k)
\end{aligned}
$$

where

$$
\begin{aligned}
& \bar{A}(p(k))=\left[\begin{array}{cc}
A & 0 \\
p(k) H(p(k)) C & G(p(k))
\end{array}\right], \bar{D}=\left[\begin{array}{c}
D \\
0
\end{array}\right], \\
& \bar{C}(p(k))=\left[\begin{array}{c}
0 \\
H(p(k)) C
\end{array}\right], \bar{B}=\left[\begin{array}{c}
B \\
0
\end{array}\right], N=\left[\begin{array}{ll}
I & 0
\end{array}\right] .
\end{aligned}
$$

Definition 1: The filtering error system (10) is said to be exponentially mean-square stable if, with $\omega(k)=0$, there exist constants $\alpha>0$ and $\tau \in(0,1)$ such that

$$
\mathbb{E}\left\{\|\bar{x}(k)\|^{2}\right\} \leq \alpha \tau^{k} \mathbb{E}\left\{\|\bar{x}(0)\|^{2}\right\}, \quad k \in \mathbb{I}^{+} .
$$

The purpose of this paper is to design a desired filter of the form (8) for the discrete nonlinear stochastic system with timevarying parameters in (1) and (6) such that, for all admissible nonlinearities, missing measurements and stochastic disturbances, the augmented system (10) is exponentially meansquare stable. 


\section{MAin Results}

In the following theorem, the parameter-dependent Lyapunov function and the convex optimization are used to deal with the stability analysis problem for the gain-scheduled filter design of the discrete-time stochastic nonlinear systems (1) and (6) with missing measurements.

Theorem 1: Consider the augmented filtering error system 10) with given filter gains. If there exist positive-definite matrix sequence $Q(p(k))>0$ and matrix $S$ such that the following matrix inequalities

$\left[\begin{array}{ccccc}-Q(p(k)) & * & * & * & * \\ F Z N & -2 I & * & * & * \\ \Omega_{1}(k) & S^{T} \bar{B} & -\Lambda(k) & * & * \\ \Omega_{2}(k) & 0 & 0 & -\Theta(k) \Lambda(k) & * \\ \sigma^{2} S^{T} \bar{D} N & 0 & 0 & 0 & -\sigma^{2} \Lambda(k)\end{array}\right]$

hold, where $\Lambda(k)=-Q(p(k+1))+S+S^{T}$ and

$$
\begin{aligned}
\Theta(k) & =p(k)(1-p(k)), \Omega_{2}(k)=\Theta(k) S^{T} \bar{C}(p(k)) N \\
\Omega_{1}(k) & =S^{T}\left[\bar{A}(p(k))+\bar{B} F_{1} Z N\right],
\end{aligned}
$$

then (10) is exponentially mean-square stable.

Proof: Define the Lyapunov function $V(k)$ := $\bar{x}^{T}(k) Q(p(k)) \bar{x}(k)$, where $Q(p(k))$ is a time-varying positive definite matrix sequence dependent on the missing probability $p(k)$. By noting $\mathbb{E}\{\xi(k)-p(k)\}=0$ and $\mathbb{E}\{\omega(k)\}=0$, it can be obtained from (10) that

$$
\begin{aligned}
\mathbb{E}\{ & \Delta V(k)\} \\
=\mathbb{E}\{[ & {[\bar{A}(p(k)) \bar{x}(k)+\bar{B} f(z(k))]^{T} Q(p(k+1))[\bar{A}(p(k)) \bar{x}(k)} \\
& +\bar{B} f(z(k))]+p(k)(1-p(k)) \bar{x}^{T}(k) N^{T} \bar{C}^{T}(p(k)) \\
& \times Q(p(k+1)) \bar{C}(p(k)) N \bar{x}(k)+\sigma^{2} \bar{x}^{T}(k) N^{T} \bar{D}^{T} \\
& \left.\times Q(p(k+1)) \bar{D} N \bar{x}(k)-\bar{x}^{T}(k) Q(p(k)) \bar{x}(k)\right\} . \quad(14)
\end{aligned}
$$

From (3) and (5), we can obtain

$$
\begin{aligned}
\mathbb{E}\{ & \Delta V(k)\} \\
\leq \mathbb{E}\{ & {\left[\left(\bar{A}(p(k))+\bar{B} F_{1} Z N\right) \bar{x}(k)+\bar{B} f_{s}(z(k))\right]^{T} } \\
& \times Q(p(k+1))\left[\left(\bar{A}(p(k))+\bar{B} F_{1} Z N\right) \bar{x}(k)\right. \\
& \left.+\bar{B} f_{s}(z(k))\right]+p(k)(1-p(k)) \bar{x}^{T}(k) N^{T} \bar{C}^{T}(p(k)) \\
& \times Q(p(k+1)) \bar{C}(p(k)) N \bar{x}(k)+\sigma^{2} \bar{x}^{T}(k) N^{T} \bar{D}^{T} \\
& \times Q(p(k+1)) \bar{D} N \bar{x}(k)-\bar{x}^{T}(k) Q(p(k)) \bar{x}(k) \\
& \left.-2 f_{s}^{T}(z(k))\left[f_{s}(z(k))+F Z N \bar{x}(k)\right]\right\} .
\end{aligned}
$$

From (14) and (15), it follows that

$$
\mathbb{E}\{\Delta V(k)\} \leq \mathbb{E}\left\{\tilde{x}^{T}(k) \Pi \tilde{x}(k)\right\},
$$

where $\tilde{x}(k)=\left[\begin{array}{ll}\bar{x}^{T}(k) & f_{s}^{T}(z(k))\end{array}\right]^{T}$ and

$$
\Pi=\left[\begin{array}{cc}
\Pi_{1} & * \\
\Pi_{2} & -2 I+\bar{B}^{T} Q(p(k+1)) \bar{B}
\end{array}\right]
$$

with

$$
\begin{aligned}
\Pi_{1}= & \left(\bar{A}(p(k))+\bar{B} F_{1} Z N\right)^{T} Q(p(k+1))\left(\bar{A}(p(k))+\bar{B} F_{1}\right. \\
& \times Z N)+\sigma^{2} N^{T} \bar{D}^{T} Q(p(k+1)) \bar{D} N+p(k)(1-p(k)) \\
& \times N^{T} \bar{C}^{T}(p(k)) Q(p(k+1)) \bar{C}(p(k)) N-Q(p(k)) \\
\Pi_{2}= & \bar{B}^{T} Q(p(k+1))\left(\bar{A}(p(k))+\bar{B} F_{1} Z N\right)+F Z N .
\end{aligned}
$$

In the following, we will conclude from (12) that $\Pi<0$. From the relation $-Q(p(k+1))+S+S^{T}>0$ in (12), we can see that $S$ is nonsingular. Performing congruence transformation $\operatorname{diag}\left\{I, I, S^{-1}, \Theta^{-1} S^{-1}, \sigma^{-2} S^{-1}\right\}$ to (12), we have

$$
\left[\begin{array}{ccccc}
-Q(p(k)) & * & * & * & * \\
F Z N & -2 I & * & * & * \\
\bar{\Omega}_{1}(k) & \bar{B} & -\bar{\Lambda}(k) & * & * \\
\bar{C}(p(k)) N & 0 & 0 & -\bar{\Theta} \bar{\Lambda}(k) & * \\
\bar{D} N & 0 & 0 & 0 & -\bar{\sigma} \bar{\Lambda}(k)
\end{array}\right]<0,
$$

with $\bar{\Lambda}(k)=-S^{-T} Q(p(k+1)) S^{-1}+S^{-1}+S^{-T}, \bar{\Omega}_{1}(k)=$ $\bar{A}(p(k))+\bar{B} F_{1} Z N, \bar{\Theta}=\Theta^{-1}(k)$ and $\bar{\sigma}=\sigma^{-2}$. Then, it $<0$, follows from inequality $S^{-T} Q(p(k+1)) S^{-1}-S^{-1}-S^{-T} \geq$ $-Q^{-1}(p(k+1))$ that

$\left[\begin{array}{ccccc}-Q(p(k)) & * & * & * & * \\ F Z N & -2 I & * & * & * \\ \bar{\Omega}_{1}(k) & \bar{B} & -\tilde{\Lambda}(k) & * & * \\ \bar{C}(p(k)) N & 0 & 0 & -\bar{\Theta} \tilde{\Lambda}(k) & * \\ \bar{D} N & 0 & 0 & 0 & -\bar{\sigma} \tilde{\Lambda}(k)\end{array}\right]<0,(20)$

with $\tilde{\Lambda}(k)=Q^{-1}(p(k+1))$. To this end, by Schur Complement Lemma, we can see that $\Pi<0$. Subsequently, we have

$$
\mathbb{E}\{\Delta V(k)\}<-\lambda_{\min }(\Pi) \mathbb{E}|\bar{x}(k)|^{2} .
$$

where $\lambda_{\min }(\Pi)$ is the minimum eigenvalue of $\Pi$ and $|\cdot|$ is the usual vector norm. Finally, we can confirm from Lemma 1 of [10] that the augmented filtering systems (10) is exponentially mean-square stable and the proof of this theorem is thus complete.

Remark 3: In Theorem 1, to improve the performance of the filter to be designed, a time-varying Lyapunov function dependent on the missing probability has been proposed. Note that, in the past few years, parameter-dependent Lyapunov functions have been intensively employed for tackling uncertain systems and time-varying parameter systems aiming to reduce the conservatism, see e.g. [1].

Remark 4: In the controller and filter design, the product terms between Lyapunov matrices and the system matrices usually have to be decoupled to bypass the difficulty encountered in the design. In this case, it is often an effective strategy to add slack variables, see e.g. [1], [5]. Along this line, in Theorem 1, we have introduced a slack variable $S$ to facilitate the resulting filter design problem.

The following theorem focuses on the design of gainscheduled filter parameters $G(p(k))$ and $H(p(k))$ according to the results in Theorem 1.

Theorem 2: Consider the discrete-time nonlinear stochastic system (1). Assume that there exist positive-definite matrix sequence $\bar{Q}(p(k))>0$, matrix sequences $\bar{H}(p(k))$ and $\bar{G}(p(k))$, nonsingular matrices $S_{11}, R_{2}$ and matrix $R_{1}$ such that the following parameter-dependent LMIs hold:

$\left[\begin{array}{ccccc}-\bar{Q}(p(k)) & * & * & * & * \\ F Z N & -2 I & * & * & * \\ \Gamma_{1}(k) & \tilde{B} & \Gamma_{2}(k) & * & * \\ \Gamma_{3}(k) & 0 & 0 & \Theta(k) \Gamma_{2}(k) & * \\ \Gamma_{4}(k) & 0 & 0 & 0 & \sigma^{2} \Gamma_{2}(k)\end{array}\right]<0$


where

$$
\begin{aligned}
& \Gamma_{1}(k)=\left[\begin{array}{ll}
S_{11}^{T} A+p(k) \bar{H}(p(k)) C+S_{11}^{T} B F_{1} Z & \bar{G}(p(k)) \\
R_{1}^{T} A+p(k) \bar{H}(p(k)) C+R_{1}^{T} B F_{1} Z & \bar{G}(p(k))
\end{array}\right] \\
& \Gamma_{2}(k)=\bar{Q}(p(k+1))-\Delta, \\
& \Gamma_{3}(k)=\Theta(k)\left[\begin{array}{c}
\bar{H}(p(k)) C \\
\bar{H}(p(k)) C
\end{array}\right] N, \Gamma_{4}(k)=\sigma^{2}\left[\begin{array}{c}
S_{11}^{T} D \\
R_{1}^{T} D
\end{array}\right] N, \\
& \Delta=\left[\begin{array}{cc}
S_{11}+S_{11}^{T} & R_{1}+R_{2}^{T} \\
R_{2}+R_{1}^{T} & R_{2}+R_{2}^{T}
\end{array}\right], \tilde{B}=\left[\begin{array}{c}
S_{11}^{T} B \\
R_{1}^{T} B
\end{array}\right] .
\end{aligned}
$$

In this case, there exist nonsingular matrices $S_{21}$ and $S_{22}$ such that $R_{2}=S_{21}^{T} S_{22}^{-T} S_{21}$, and then the gains of the desired filter can be obtained as follows:

$G(p(k))=S_{21}^{-T} \bar{G}(p(k)) S_{21}^{-1} S_{22}, H(p(k))=S_{21}^{-T} \bar{H}(p(k))$.

Then, there exists a desired gain-scheduled filter in the form of (8) such that the filtering error dynamics (10) is exponentially mean-square stable.

Proof: Let the nonsingular matrix variable $S$ in (12) be partitioned as $S=\left[S_{i j}\right]_{2 \times 2}$ where $S_{11}, S_{21}$ and $S_{22}$ are nonsingular matrices. Introduce matrices

$$
\begin{gathered}
\mathcal{T}=\left[\begin{array}{cc}
I & 0 \\
0 & S_{22}^{-1} S_{21}
\end{array}\right], \bar{Q}(p(k))=\mathcal{T}^{T} Q(p(k)) \mathcal{T}, \\
R_{1}=S_{12} S_{22}^{-1} S_{21}, R_{2}=S_{21}^{T} S_{22}^{-T} S_{21} .
\end{gathered}
$$

By congruence transformation $\operatorname{diag}\left\{\mathcal{T}^{-1}, I, \mathcal{T}^{-1}, \mathcal{T}^{-1}, \mathcal{T}^{-1}\right\}$, we can see that (22) is equivalent to (12), and it then follows from Theorem 1 that (10) is exponentially mean-square stable.

Apparently, the number of LMIs in Theorem 2 is actually infinite due to the time-varying parameter $p(k)$ and, therefore, it is nearly impossible to solve the LMIs directly. In the following, we will convert the LMIs into finite ones.

Theorem 3: For system (1), assume that there exist positive positive-definite matrices $\bar{Q}_{0}>0$ and $\bar{Q}_{p}>0$, nonsingular matrices $S_{11}, R_{2}$ and matrices $R_{1}, \bar{G}_{0}, \bar{G}_{f}, \bar{H}_{0}$ and $\bar{H}_{f}$ such that the following LMIs hold:

$$
\mathbb{M}^{i j r l}=\left[\begin{array}{ccccc}
-\bar{Q}^{i} & * & * & * & * \\
F B N & -2 I & * & * & * \\
\Gamma_{1}^{i j} & \tilde{B} & \Gamma_{2}^{l} & * & * \\
\Gamma_{3}^{i j r} & 0 & 0 & \bar{\Theta}^{j r} \Gamma_{2}^{l} & * \\
\Gamma_{4} & 0 & 0 & 0 & \sigma^{2} \Gamma_{2}^{l}
\end{array}\right]<0,
$$

for $i, j, r, l=1,2$, where $\Gamma_{5}$ and $\tilde{B}$ have been defined in (22)

$$
\begin{aligned}
& \Gamma_{1}^{i j}=\left[\begin{array}{ll}
S_{11}^{T} A+p_{j}\left(\bar{H}_{0}+p_{i} \bar{H}_{f}\right) C+S_{11}^{T} B F_{1} Z & \bar{G}^{i} \\
R_{1}^{T} A+p_{j}\left(\bar{H}_{0}+p_{i} \bar{H}_{f}\right) C+R_{1}^{T} B F_{1} Z & \bar{G}^{i}
\end{array}\right], \\
& \Delta=\left[\begin{array}{cc}
S_{11}+S_{11}^{T} & R_{1}+R_{2}^{T} \\
R_{2}+R_{1}^{T} & R_{2}+R_{2}^{T}
\end{array}\right], \Gamma_{3}^{i j r}=\bar{\Theta}^{j r}\left[\begin{array}{c}
\bar{H}^{i} C \\
\bar{H}^{i} C
\end{array}\right] N \\
& \bar{Q}^{i}=\bar{Q}_{0}-p_{i} \bar{Q}_{p}, \Gamma_{2}^{l}=\bar{Q}^{l}-\Delta, \bar{\Theta}^{j r}=p_{j}\left(1-p_{r}\right),, \\
& \bar{H}^{i}=\bar{H}_{0}+p_{i} \bar{H}_{f}, \bar{G}^{i}=\bar{G}_{0}+p_{i} \bar{G}_{f} \text {. }
\end{aligned}
$$

In this case, there exist nonsingular matrices $S_{21}$ and $S_{22}$ such that $R_{2}=S_{21}^{T} S_{22}^{-T} S_{21}$, and therefore the constant filter gains are obtained as follows:

$$
\begin{aligned}
& G_{0}=S_{21}^{-T} \bar{G}_{0} S_{21}^{-1} S_{22}, G_{f}=S_{21}^{-T} \bar{G}_{f} S_{21}^{-1} S_{22} \\
& H_{0}=S_{21}^{-T} \bar{H}_{0}, H_{f}=S_{21}^{-T} \bar{H}_{f} .
\end{aligned}
$$

Then, a gain-scheduled filter can be obtained in the form of (8) such that the filtering error dynamics (10) is exponentially mean-square stable.

Proof: Firstly, choose the probability-dependent Lyapunov matrices as

$$
Q(p(k))=Q_{0}+p(k) Q_{p}
$$

where $Q_{0}>0$ and $Q_{p}>0$. It is easily seen that $\bar{Q}(p(k))=$ $\bar{Q}_{0}+p(k) \bar{Q}_{p}$ with $\bar{Q}_{0}=\mathcal{T}^{T} Q_{0} \mathcal{T}$ and $\bar{Q}_{p}=\mathcal{T}^{T} Q_{p} \mathcal{T}$.

Setting

$$
\alpha_{1}(k)=\frac{p_{2}-p(k)}{p_{2}-p_{1}}, \quad \alpha_{2}(k)=\frac{p(k)-p_{1}}{p_{2}-p_{1}},
$$

we have

$$
\left\{\begin{array}{l}
\alpha_{1}(k)+\alpha_{2}(k)=1, \alpha_{i}(k) \geq 0(i=1,2) \\
p(k)=\alpha_{1}(k) p_{1}+\alpha_{2}(k) p_{2}
\end{array}\right.
$$

Similarly, letting

$$
\beta_{1}(k)=\frac{p_{2}-p(k+1)}{p_{2}-p_{1}}, \quad \beta_{2}(k)=\frac{p(k+1)-p_{1}}{p_{2}-p_{1}},
$$

we have

$$
\left\{\begin{array}{l}
\beta_{1}(k)+\beta_{2}(k)=1, \beta_{l}(k) \geq 0(l=1,2) \\
p(k+1)=\beta_{1}(k) p_{1}+\beta_{2}(k) p_{2} .
\end{array}\right.
$$

From the above transformations, it is easily derived that

$$
\begin{gathered}
\bar{Q}(p(k))=\sum_{i=1}^{2} \alpha_{i}(k) \bar{Q}^{i}, \quad \bar{Q}(p(k+1))=\sum_{l=1}^{2} \beta_{l}(k) \bar{Q}^{l}, \\
\bar{G}(p(k))=\sum_{i=1}^{2} \alpha_{i}(k) \bar{G}^{i}, \quad \bar{H}(p(k))=\sum_{i=1}^{2} \alpha_{i}(k) \bar{H}^{i} .
\end{gathered}
$$

Furthermore, it follows from (24) that

$$
\sum_{i, j, r, l=1}^{2} \alpha_{i}(k) \alpha_{j}(k) \alpha_{r}(k) \beta_{l}(k) \mathbb{M}^{i j r l}<0 .
$$

Also, it follows from (29) and (31)-(33) that (22) holds. The proof is now complete.

In Theorem 3, we convert infinite LMIs in Theorem 2 to finite ones by turning the time-varying parameter $p(k)$ into the polytopic form. By such a transformation, the constant gains of the desired gain-scheduled filter can be easily derived in terms of the available LMI toolbox by using the computationally appealing gain-scheduled filter design algorithm listed as follows.

Algorithm 1: The gain-scheduled filter design algorithm.

Step 1: Given the initial values for the positive integer $N$, the initial state $\rho$, the constants $p_{1}$ and $p_{2}$, the matrices $A, B$, $C, D, F_{1}, F_{2}$ and $Z$, select appropriate initial state estimate $\rho_{f}$ and set $k=0$.

Step 2: Solve the LMI in (24) to obtain the positive-definite matrices $\bar{Q}_{0}$ and $\bar{Q}_{p}$, matrices $R_{2}, \bar{G}_{0}, \bar{G}_{f}, \bar{H}_{0}$ and $\bar{H}_{f}$. Choose appropriate nonsingular matrices $S_{21}$ and $S_{22}$ to derive the constant filter parameters $G_{0}, G_{f}, H_{0}$ and $H_{f}$ by (26).

Step 3: Based on the measured time-varying parameter $p(k)$, compute the filter gains $G(p(k))$ and $H(p(k))$ by (9) and the state estimate $x_{f}(k+1)$ by (8). Then, set $k=k+1$.

Step 4: If $k<N$, go to Step 3, otherwise go to Step 5. 


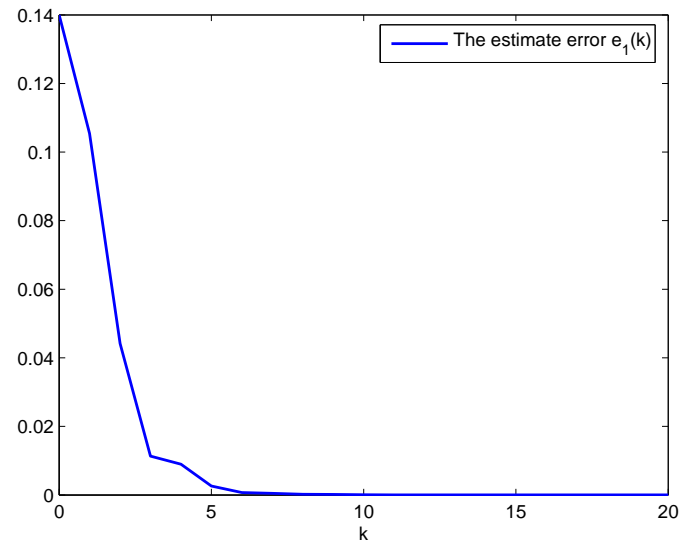

Fig. 1. Estimate error $e_{1}(k)$

\section{Step 5: Stop.}

Remark 5: Our main results are based on the LMI conditions. While the interior-point LMI solvers are significantly faster than classical convex optimization algorithms, it should be kept in mind that the complexity of LMI computations remains higher than that of solving, say, a Riccati equation. For instance, problems with a thousand design variables typically take over an hour on today's workstations. However, research on LMI optimization is a very active area in the applied math, optimization and the operations research community, and substantial speed-ups can be expected in the future.

\section{AN ILlustrative EXAMPLE}

The system parameters of (1) and (6) are given as follows

$$
\begin{gathered}
A=\left[\begin{array}{cc}
0.43 & 0 \\
0.15 & 0.36
\end{array}\right], B=\left[\begin{array}{cc}
0.1 & 0.04 \\
0 & 0.08
\end{array}\right], p_{1}=0.4, p_{2}=0.8, \\
D=\left[\begin{array}{cc}
0.3 & 0.03 \\
0.05 & 0.38
\end{array}\right], Z=\left[\begin{array}{cc}
0.51 & 0 \\
0 & 0.621
\end{array}\right], C=\left[\begin{array}{ll}
0.38 & 0.46
\end{array}\right], \\
F_{1}=\operatorname{diag}\{0.460 .37\}, F_{2}=\operatorname{diag}\{2.812 .95\}, \sigma^{2}=1 .
\end{gathered}
$$

Assume that the measurable missing probability sequence satisfies $p(k)=p_{1}+\left(p_{2}-p_{1}\right)|\sin (k)|$. According to Theorem 3 and Algorithm 1, the constant filter parameters $G_{0}, G_{f}, H_{0}$ and $H_{f}$ can be obtained as follows:

$$
\begin{gathered}
G_{0}=\left[\begin{array}{cc}
0.0242 & -0.0007 \\
0.0086 & 0.0140
\end{array}\right], H_{0}=\left[\begin{array}{c}
-1.3778 \\
-1.5091
\end{array}\right], \\
G_{f}=\left[\begin{array}{cc}
0.0030 & 0.0084 \\
0.0070 & 0.0059
\end{array}\right], H_{f}=\left[\begin{array}{l}
0.9806 \\
1.0835
\end{array}\right] .
\end{gathered}
$$

With the available missing probability $p(k)$, the gainscheduled filter gains $G(p(k))$ and $H(p(k))$ and the state estimate $x_{f}(k)$ can be obtained. Figs. 1-2 show the estimate errors $e_{1}(k)=x_{1}(k)-x_{f 1}(k)$ and $e_{2}(k)=x_{2}(k)-x_{f 2}(k)$, respectively. The simulation results have illustrated our theoretical analysis.

\section{REFERENCES}

[1] H. Gao, X. Meng and T. Chen, A parameter-dependent approach to robust $H_{\infty}$ filtering for time-delay systems, IEEE Trans. Automatic Control, Vol. 53, No. 10, pp. 2420-2425, 2008

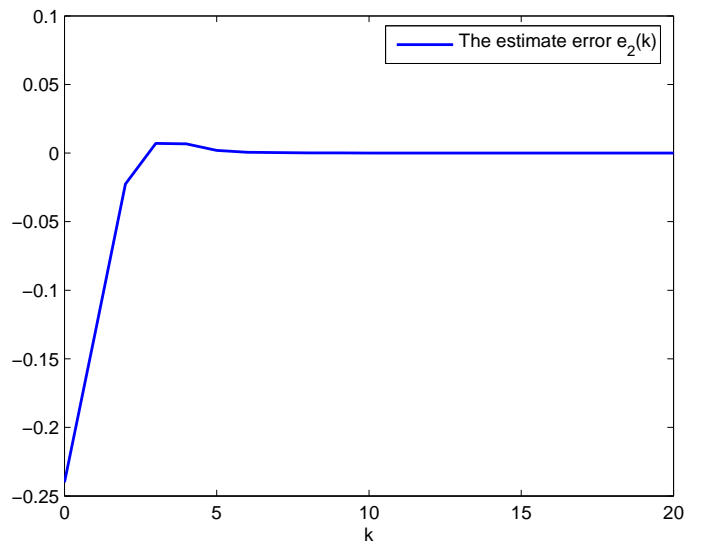

Fig. 2. Estimate error $e_{2}(k)$

[2] H. Gao, J. Lam and C. Wang, Mixed $H_{2} / H_{\infty}$ filtering for continuoustime polytopic systems: a parameter-dependent approach, circuits, Systems and Signal Processing, Vol. 24, No. 6, pp. 689-702, 2005.

[3] H. Gao, J. Lam, L. Xie and C. Wang, New approach to mixed $H_{2} / H_{\infty}$ filtering for polytopic discrete-time systems, IEEE Trans. Signal Processing, Vol. 53, No. 8, pp. 3183-3192, 2005

[4] H. Gao, J. Lam, P. Shi and C. Wang, Parameter-dependent filter design with guaranteed H-infinity performance, IEE Proc. Control Tjeory and Applications, Vol. 152, No. 5, pp. 531-537, 2005.

[5] N. Hoang, H. Tuan, P. Apkarian and S. Hosoe, Gain-scheduled filtering for time-varying discrete systems, IEEE Tran. Signal Processing, Vol. 52, No. 9, pp. 2464-2476, 2004

[6] J. Lam, H. Gao, S. Xu and C. Wang, $H_{\infty}$ and $L_{2} / L_{\infty}$ model reduction for system input with sector nonlinearities, J. Optim. Theory and Appl., Vol. 125, No. 1, pp. 137-155, 2005.

[7] G.-P. Liu, Predictive controller design of networked systems with communication delays and data loss, IEEE Trans. Circuits and Systems -II: Express Briefs, Vol. 57, No. 6, pp. 481-485, 2010.

[8] X. Meng, J. Lam and Z. Fei, A generalized parameter-dependent approach to robust $H_{\infty}$ filtering of stochastic systems, circuits, Systems and Signal Processing, Vol. 28, No. 2, pp. 191-204, 2009.

[9] S. Sun, L. Xie, W. Xiao and N. Xiao, Optimal filtering for systems with multiple packet dropouts, IEEE Trans. Circuits and Systems -II: Express Briefs, Vol. 55, No. 7, pp. 695-699, 2008.

[10] Z. Wang, F. Yang, D.W.C. Ho, and X. Liu, Robust $H_{\infty}$ filtering for stochastic time-delay systems with missing measurements, IEEE Trans. Signal Processing, Vol. 54, No. 7, pp. 2579-2587, 2006.

[11] Z. Wang, Y. Liu and X. Liu, $H_{\infty}$ filtering for uncertain stochastic timedelay systems with sector-bounded nonlinearities, Automatica, Vol. 44, No. 5, pp. 1268-1277, 2008.

[12] G. Wei, Z. Wang, X. He and H. Shu, Filtering for networked stochastic time-delay systems with sector nonlinearity, IEEE Tran. Circuits and Systems - Part II, Vol. 56, No. 1, pp. 71-75, 2009.

[13] J. Wilson, A. Nelson and B. Farhang-Boroujeny, Parameter derivation of type-2 discrete-time phase-locked loops containing feedback delays, IEEE Tran. Circuits and Systems - Part II, Vol. 56, pp. 886-890, Dec. 2009.

[14] J. Zhang, Y. Xia and P. Shi, Parameter-dependent robust $H_{\infty}$ filtering for uncertain discrete-time systems, Automatica, Vol. 45, no. 2, pp. 560565,2009

[15] L. Zhang, P. Shi, E.-K. Boukas and C. Wang, Robust $l_{2}-l_{\infty}$ filtering for switched linear discrete time-delay systems with polytopic uncertainties, IET Control Theory and Applications, Vol. 1, No. 3, pp. 722-730, 2007.

[16] S. Zhou, B. Zhang and W.-X. Zheng, Gain-scheduled $H_{\infty}$ filtering of parameter-varying discrete-time systems via parameter-dependent Lyapunov functions, Int. J. Control, Automation and Systems, Vol. 7, No. 3, pp. 475-479, 2009. 